\title{
RESEARCH ON MODELING AND COMPENSATION METHOD FOR GROUND LEVEL ERROR BASED ON MOBILE MANIPULATOR
}

\author{
Shuai GUO', Chengtao YUE 2, Tao SONG ${ }^{3}$, Qibo JIA ${ }^{4}$ \\ 1,23,4 Shanghai Key Laboratory of Intelligent Manufacturing and Robotics, School of Mechatronics, Engineering and \\ Automation, Shanghai University, HC204, No. 99, Road Shangda, Shanghai 200444, China \\ ${ }^{1}$ Shanghai Robot Industrial Technology Research Institute, 509 Wuning Road, Putuo District, Shanghai, China \\ guoshuai@shu.edu.cn, yctao@shu.edu.cn, songtao43467226@shu.edu.cn \\ 1731977106@qq.com
}

\begin{abstract}
In this paper, a modeling and compensation method for ground level error based on mobile manipulator is proposed. The ground level error is caused by the former civil construction and will affect the accuracy of baseline drawing and subsequent decoration engineering. This paper focuses on a mobile manipulator which can carry out decoration engineering automatically. The ground level error should be dealt with before baseline drawing. The entire method can be summed up into three steps. The first step is spatial kinematics modeling for mobile manipulator. The second one is step ground level error acquisition with tilt sensor mounted on the system. The last one is curve fitting and compensation based on the mobile manipulator. Two fitting methods are proposed: direct polynomial fitting and piecewise polynomial fitting which aims to common baseline and high-grade baseline respectively. The experiment was also carried out to verify the proposed method.
\end{abstract}

Keywords: Mobile Manipulator, Ground Level Error, Compensation.

\section{Introduction}

A mobile manipulator with extended mobility can be utilized in various applications, such as drilling/riveting in aircraft manufacturing, nuclear reactor maintenance [1-3]. In recent years, automation, especially robot-based automation, is increasingly prevalent in the domain of construction industry. Most of the tasks in this domain are based on manual operation supported by machineries, such as hoists and cranes or by hand-held tools, which often are of considerable weight and which expose the worker to high levels of dust, noise, and vibration. In order to adapt to more complex environments of construction, mobile systems are used widely. In situ Fabricator developed by ETH Zürich could build a full-size undulating brick wall and be use to fabricate mesh module in the concreting process [4]. The robot system used for the automated removal of asbestos contamination was developed by RWTH Aachen University in Bots2Rec project [5]. Cable-Driven-Parallel-Robots developed by Jean-Baptiste Izard combined the extruder and the Cogiro CDPR fabricates 3D prints [6]. The ROBO-SPECT Robotic platform developed by Universidad Carlos III De Madrid inspected tunnels automatically [7]. Massachusetts Institute of Technology presented the Digital Construction Platform which was an automated construction system capable of customized on-site fabrication of architectural-scale structures using real-time environmental data for process control [8].

There are several issues associated with mobile manipulator [9-10]. The modeling and compensation of error is one of them which attracted the attention of researchers. The decouple method proposed by Y. Y. Hsu et al. [11] was for geometry errors of five-axis machine tools. F. F. Xi et al. [12] presented a method to improve inspection accuracy of 3D line laser through error characterization. Myeong-Woo Cho et al. [13] proposed an integrated machining error compensation method based on polynomial neural network for improving the accuracy of the onmachine measurement system. Jinhyun Kim et al. [14] proposed a method using zero moment point compensation for dynamic stability of a mobile manipulator. N. A. Duffie et al. [15] proposed a compensation method to obtain values of coefficients in a kinematic model for mechanical or software error correction. Changhai Ru et al. [16] proposed a method for piezoelectric actuator based on a PID feedback controller with a feedforward compensation. Jianjun Wang et al. [17] presented a method to compensate the robot deformation caused by the machining force. Chung-Lin Huang and ChaoYuen Hsu [18] proposed a compensation method called Hierarchical Grid Interpolation for motion estimation. Xinda Song et al. [19] proposed a method for compensating the estimated back electromotive force harmonic error efficiently and adaptively. John 
M. Fines et al. [20] proposed a method using artificial neural networks for positioning error compensation. Takaaki Oiwa et al. [21] proposed a compensation method for elastic and thermal deformations of the machine frame supporting the mechanism. However, modeling and compensation issue for ground level error is not fully solved.

\section{Problem Formulation}

In the architectural decoration, the foundational task is to base-line drawing which transfers drawings to the actual scene and giving annotations, markings and drawings according to the measurement data. The contents marked on the drawings are accurately laid out on the floor, walls and ceiling in a scale of $1: 1$ at the construction site. In China, a horizontal reference line $(1.0 \mathrm{~m}$ line) will be first derived from the construction's elevation point. Then the other baselines will be drawn, including vertical/horizontal controlling line, line for localization, representing line of various finished works, constructing line for concealed work. These lines can be classified into two types. One is common baseline with common accuracy needed, usually 5 $\mathrm{mm}$. This type usually includes constructing line for concealed work. The other is high-grade base line with high accuracy needed, usually $3 \mathrm{~mm}$ which includes other baselines. Baseline drawing can effectively reduce the dimensional error in the process of building construction.

Baseline drawing also affect the amount of materials used in the construction process which will reduce energy consumption and pollution. It also can solve the internal coordination problems in the decoration project and eliminate the contradiction of the construction of various professional. In sum, baseline drawing directly affects the final effect of decoration engineering.

Through investigation, it can be found that the artificial baseline drawing has high technical requirements for construction workers which causes low work efficiency. MoMaCo (Mobile Manipulator for Construction) developed by the Shanghai University is used for the automatically baseline drawing at construction site, as shown in Fig. 1 (a). MoMaCo can adapts to the indoor working environment in the construction field and completes the baseline drawing task. It should be noticed that the ground before decoration is not flat which is caused by civil construction. The ground error of horizontality, straightness and flatness will lead to the failure of baseline drawing. This problem should be dealt with in the process of baseline drawing with MoMaCo. After drawing, the indoor environment is as shown in Fig. 1 (b).
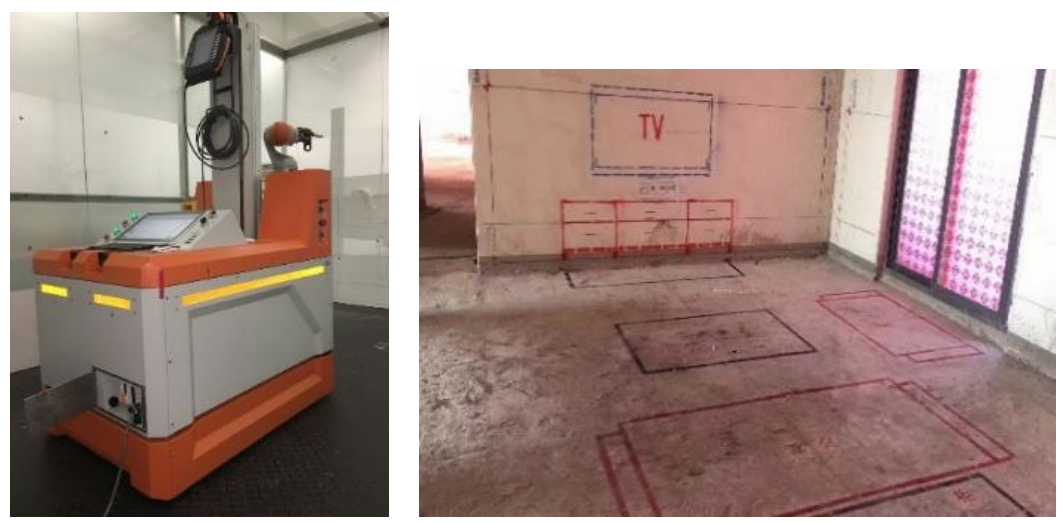

(a)

(b)

Figure 1: (a) MoMaCo (b) The Ground Before Decoration on Construction Site

\section{System Modeling}

\subsection{Function of MoMaCo}

As shown in Figure 2, MoMaCo can move omnidirectionally in the space with localization and navigation function which can cover the drawing operation with a height of $2700 \mathrm{~mm}$. It also has forcecontrol and obstacle detection function which can protect human being around it. The spatial mobile platform of MoMaCo consists of an omnidirectional mobile platform drove by four Mecanum wheels and a vertical slider which can realize front-rear, leftright, up-down and zero radius rotation movement in the space. This spatial mobile platform ensures that the system can reach the target position more flexibly and quickly. The manipulator uses KUKA LBR iiwa which is with 7 DOF. The tip of the manipulator is equipped with a baseline drawing tool. The localization and navigation unit is composed of two laser range finders of LMS100 from SICK, with which the position and pose of MoMaCo can be obtained. MoMaCo also equips a TMM88A- 
PCI090 tilt sensor from SICK which can measure the tilt angles of the system in two dimensions relative to the horizontal ground.

As shown in Figure 2, when MoMaCo draws baselines in the indoor environment, the global frame $\left\{\mathrm{X}_{\mathrm{G}}, \mathrm{Y}_{\mathrm{G}}, \mathrm{Z}_{\mathrm{G}}\right\}$ is attach to the corner of the wall for MoMaCo's localization and navigation. MoMaCo's local frame $\left\{\mathrm{X}_{\mathrm{L}}, \mathrm{Y}_{\mathrm{L}}, \mathrm{Z}_{\mathrm{L}}\right\}$ is attach to the center point of the system and determines the direction in which the robot move omni-directionally in the space. The manipulator's base frame $\left\{\mathrm{X}_{\mathrm{B}}, \mathrm{Y}_{\mathrm{B}}, \mathrm{Z}_{\mathrm{B}}\right\}$ is attach to the lifting slider which moves up and down. The tool frame $\left\{\mathrm{X}_{\mathrm{M}}, \mathrm{Y}_{\mathrm{M}}, \mathrm{Z}_{\mathrm{M}}\right\}$ is attach to the tool of manipulator.

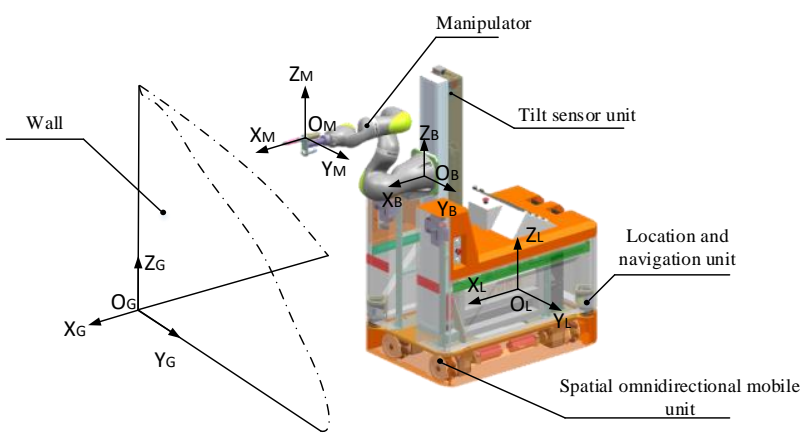

Figure 2 : Coordinates frames of MoMaCo

\subsection{Spatial kinematics modeling}

There are 4 DOFs in the spatial mobile platform as shown in Figure 3, i.e. the translation in $\left\{\mathrm{X}_{\mathrm{G}}, \mathrm{Y}_{\mathrm{G}}, \mathrm{Z}_{\mathrm{G}}\right\}$ and rotation in $Z_{-} G$ direction. Such platform can make the base of manipulator move to the most indoor space. With the reachability of manipulator, the system can draw baselines in the walls, floor and the ceiling.

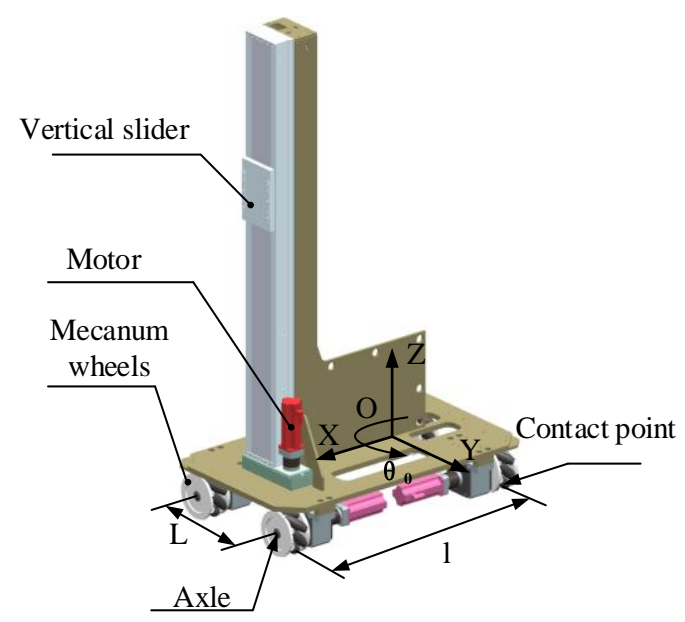

Figure 3 : Spatial omnidirectional mobile unit
According to the kinematics analysis [22-23], the motion equation of the system can be obtained as

$$
\begin{gathered}
\overrightarrow{\mathbf{V}}=\mathrm{D} \overrightarrow{\mathrm{W}} \\
\text { Where } \overrightarrow{\mathbf{V}}=\left[\begin{array}{c}
\mathrm{V}_{\mathrm{X}} \\
\mathrm{V}_{\mathrm{Y}} \\
\mathrm{W}_{0} \\
\mathrm{~V}_{\mathrm{Z}}
\end{array}\right], \\
\mathrm{D}=\frac{1}{4}\left[\begin{array}{ccccc}
-\mathrm{R} & \mathrm{R} & -\mathrm{R} & \mathrm{R} & 0 \\
\frac{\mathrm{R}}{\mathrm{R}} & \mathrm{R} & \mathrm{R} & \mathrm{R} & 0 \\
\frac{\mathrm{R}+\mathrm{L}}{\mathrm{L}} & -\frac{\mathrm{R}}{1+\mathrm{L}} & -\frac{\mathrm{R}}{1+\mathrm{L}} & \frac{\mathrm{R}}{\mathrm{I}+\mathrm{L}} & 0 \\
0 & 0 & 0 & 0 & \frac{\mathrm{Ph}}{30 \pi}
\end{array}\right], \overrightarrow{\mathrm{W}}=\left[\begin{array}{c}
\mathrm{w} 1 \\
\mathrm{w} 2 \\
\mathrm{w} 3 \\
\mathrm{w} 4 \\
\mathrm{w} 5
\end{array}\right] .
\end{gathered}
$$

Where $\vec{V}$ is the velocity of the system with $\left[\mathrm{V}_{\mathrm{X}}, \mathrm{V}_{\mathrm{Y}}, \mathrm{V}_{\mathrm{Z}}\right]^{\mathrm{T}}$ representing the velocity component in three directions and $W_{0}$ representing the velocity component in $\mathrm{Z}_{\mathrm{L}}$ direction. $\mathrm{L}$ is half of the distance from the front axle to the rear axle. 1 is the transverse distance from the wheel centers which also are the contact points on the ground to the platform center line. $\mathrm{R}$ is the radius of four wheels. $\mathrm{w} 1, \mathrm{w} 2, \mathrm{w} 3, \mathrm{w} 4$ are angular velocity of the four wheels respectively, and $w 5$ is angular velocity of lift motor. $\mathrm{Ph}$ is the lead of the ball screw.

MoMaCo first moves the tool to contact with the wall and then moves the spatial mobile platform and manipulator simultaneously to draw the baseline. The position of the manipulator will be affected by the ground level error which results in nonstraightness of the baseline. This error will lead to the failure of baseline drawing and should be eliminated.

\section{Error Modeling and Compensation}

In consideration of the difference of the ground level error in different environment, it should be sensed, modeled and then compensated before baseline drawing with MoMaCo.

\subsection{Step Modeling of ground level error}

MoMaCo moves step by step in the indoor environment, and the step distance is set as $150 \mathrm{~mm}$ which is half the width of the spatial mobile platform. The position/posture set $\left(\mathrm{X}, \mathrm{Y}_{v} \mathrm{Z}_{v}, \theta, \alpha, \beta\right)$ can be acquired at each position, where $(X, Y, Z, \theta)$ is the localization data, and $(\alpha, \beta)$ is the tilt angle set from the tilt sensor. 


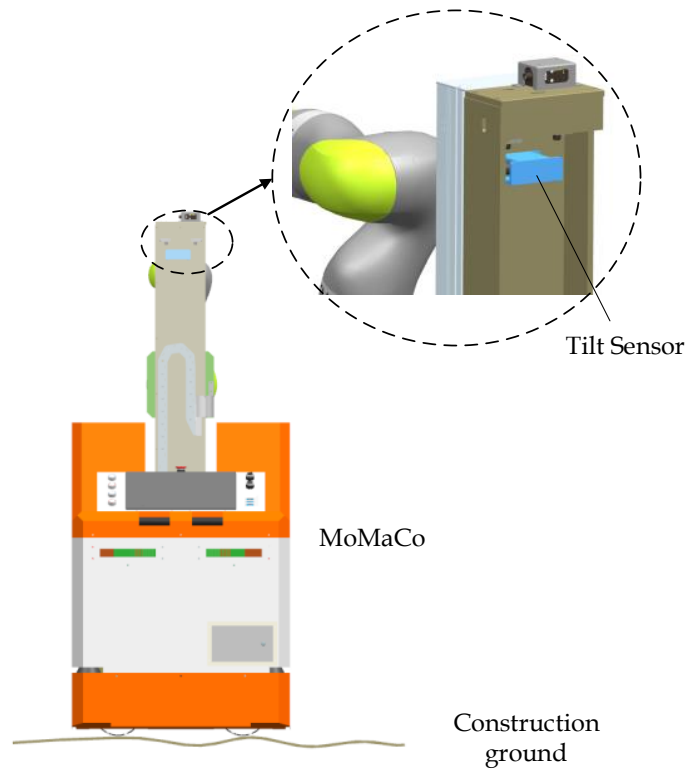

Figure 4: MoMaCo and the tilt sensor

As shown in Figure 4, the tilt sensor mounted on the system will transfer the tilt angles data to MoMaCo all the times. And as shown in Figure 5, when MoMaCo stands in one position, ground level error e in each step can be computed from step distance $\mathrm{H}$ and tilt angle given in Table 1 . When MoMaCo moves around in the environment step by step, the ground level error of the whole floor can then be obtained with serial of step errors.

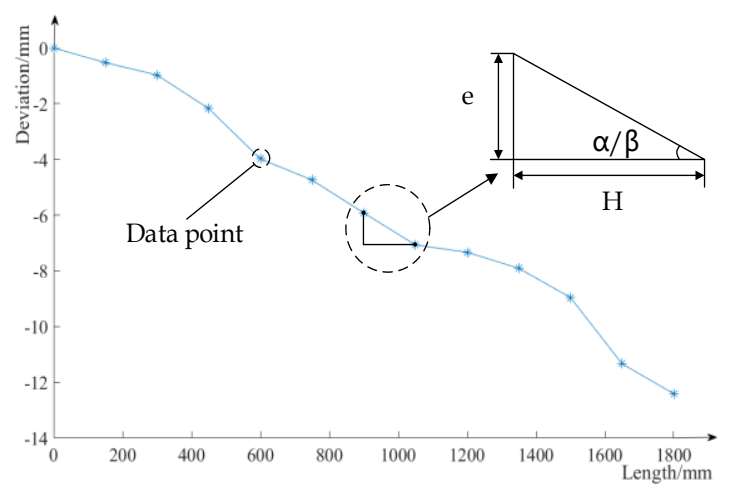

Figure 5 : Step ground level error
Table 1. A Group of Tilt Angle and Step Ground Level

Error

\begin{tabular}{|c|c|c|}
\hline No. & Tilt Angle $^{\circ}$ ) & $\begin{array}{c}\text { Step Ground Level } \\
\text { Error(mm) }\end{array}$ \\
\hline 1 & -0.20 & -0.52 \\
\hline 2 & -0.17 & -0.45 \\
\hline 3 & -0.46 & -1.20 \\
\hline 4 & -0.69 & -1.81 \\
\hline 5 & -0.29 & -0.76 \\
\hline 6 & -0.45 & -1.18 \\
\hline 7 & -0.44 & -1.15 \\
\hline 8 & -0.10 & -0.26 \\
\hline 9 & -0.22 & -0.58 \\
\hline 10 & -0.40 & -1.05 \\
\hline 11 & -0.91 & -2.38 \\
\hline 12 & -0.41 & -1.07 \\
\hline 13 & -0.41 & $/$ \\
\hline
\end{tabular}

\subsection{Direct polynomial fitting}

The serial of step errors cannot be used for compensation directly because there are cuspidal point between adjacency step. This cuspidal point will cause the abrupt acceleration in corresponding direction of MoMaCo which is not suitable for the baseline drawing, even leading to the failure of accuracy. For trajectory planning in the next step, the fitting method is proposed here to deal with the serial of step errors.

The fitting curve using cubic polynomial like $y=a x^{3}+b x^{2}+c x+d$. The Eq. (2) should be met with $[\mathrm{a}, \mathrm{b}, \mathrm{c}, \mathrm{d}]$ obtained.

$$
\arg \min _{[a, b, c, d]}\left(S_{D P}\right)
$$

Where $S_{D P}=\sum_{i=1}^{n}\left(a x_{i}^{3}+b x_{i}^{2}+c x_{i}+d-y_{i}\right)^{2}$. The partial derivative as shown in Eq. (3) is used for the solution.

$$
\frac{\partial S_{D P}}{\partial a}=0, \frac{\partial S_{D P}}{\partial b}=0, \frac{\partial S_{D P}}{\partial c}=0, \frac{\partial S_{D P}}{\partial d}=0
$$

Eq. (4) can be obtained from Eq. (3).

$$
\left\{\begin{array}{c}
2 \sum_{i=1}^{n}\left(a x_{i}^{3}+b x_{i}^{2}+c x_{i}+d-y_{i}\right)=0 \\
2 \sum_{i=1}^{n}\left(a x_{i}^{3}+b x_{i}^{2}+c x_{i}+d-y_{i}\right) \cdot x_{i}=0 \\
2 \sum_{i=1}^{n}\left(a x_{i}^{3}+b x_{i}^{2}+c x_{i}+d-y_{i}\right) \cdot x_{i}^{2}=0 \\
2 \sum_{i=1}^{n}\left(a x_{i}^{3}+b x_{i}^{2}+c x_{i}+d-y_{i}\right) \cdot x_{i}^{3}=0
\end{array}\right.
$$

Then, Eq. (5) can be obtained with Eq. (4) simplified, 


$$
\left\{\begin{array}{c}
a \sum_{i=1}^{n} x_{i}^{3}+b \sum_{i=1}^{n} x_{i}^{2}+c \sum_{i=1}^{n} x_{i}+n d=\sum_{i=1}^{n} y_{i} \\
a \sum_{i=1}^{n} x_{i}^{4}+b \sum_{i=1}^{n} x_{i}^{3}+c \sum_{i=1}^{n} x_{i}^{2}+d \sum_{i=1}^{n} x_{i}=\sum_{i=1}^{n}\left(y_{i} \cdot x_{i}\right) \\
a \sum_{i=1}^{n} x_{i}^{5}+b \sum_{i=1}^{n} x_{i}^{4}+c \sum_{i=1}^{n} x_{i}^{3}+d \sum_{i=1}^{n} x_{i}^{2}=\sum_{i=1}^{n}\left(y_{i} \cdot x_{i}^{2}\right) \\
a \sum_{i=1}^{n} x_{i}^{6}+b \sum_{i=1}^{n} x_{i}^{5}+c \sum_{i=1}^{n} x_{i}^{4}+d_{1} \sum_{i=1}^{n} x_{i}^{3}=\sum_{i=1}^{n}\left(y_{i} \cdot x_{i}^{3}\right)
\end{array}\right.
$$

Eq. (5) can be represented by Eq. (6) in matrix form,

$$
\mathbf{Y}=\mathbf{X} \cdot \mathbf{C}
$$

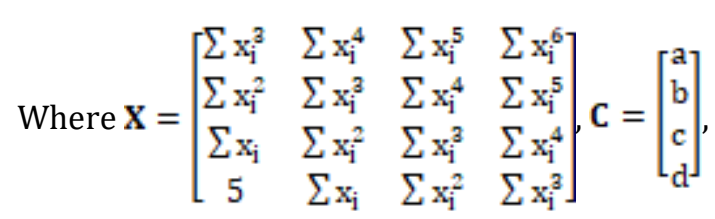

$$
\begin{aligned}
& \mathbf{Y}=\left[\begin{array}{c}
\Sigma\left(\mathrm{y}_{\mathrm{i}} \cdot \mathrm{x}_{\mathrm{i}}^{\mathrm{a}}\right) \\
\Sigma\left(\mathrm{y}_{\mathrm{i}} \cdot \mathrm{x}_{\mathrm{i}}^{2}\right) \\
\Sigma\left(\mathrm{y}_{\mathrm{i}} \cdot \mathrm{x}_{\mathrm{i}}\right) \\
\Sigma \mathrm{y}_{\mathrm{i}}
\end{array}\right]
\end{aligned}
$$

C can be determined with Eq. (7)

$$
\mathbf{C}=\left(\mathbf{X}^{\mathrm{T}} \mathbf{X}\right)^{-1} \mathbf{X}^{\mathrm{T}} \mathbf{Y}
$$

The fitting error (E) can be obtained as the distance from the data point to the fitted curve. The fitting error can be further decreased if the fiveorder polynomial fitting method can be used, but it will cause the complexity of control. Technically, to guarantee the accuracy of the baseline, the fitting error of common baseline should be controlled under $1 \mathrm{~mm}$ while the fitting error of high-grade baseline should be controlled under $0.5 \mathrm{~mm}$. For the most circumstances, direct polynomial fitting is enough for error modelling. With the data given in Table 1 , the fitting results are: $\mathrm{C}=[-8.5902 \mathrm{e}-10,1.7979 \mathrm{e}-$ $6,-0.0075,0.4491]^{\mathrm{T}}$

\section{with Eq. (7).}

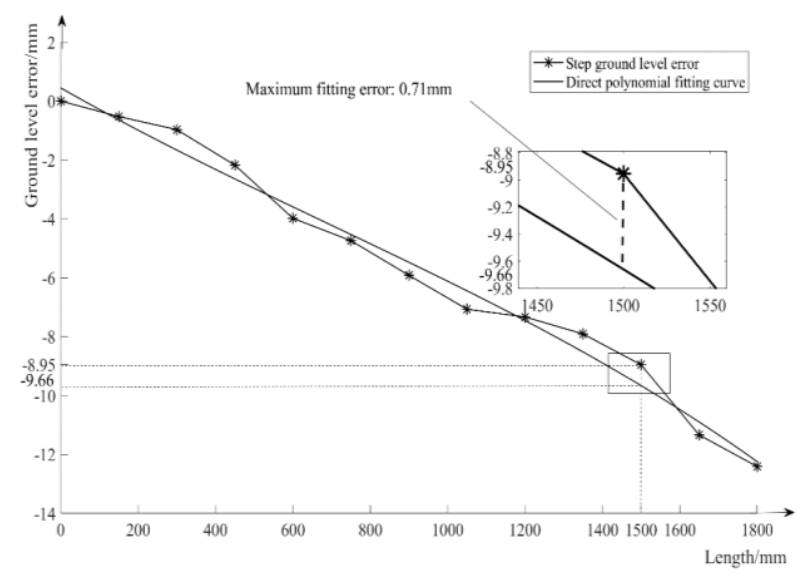

Figure 6: Direct Polynomial Fitting
According to the Figure 6, the maximum fitting error occurred while the length was $1,500 \mathrm{~mm}$. At this point the fitting error was $0.71 \mathrm{~mm}$ which can meet the need of fitting error of common baseline. It should be noticed that $\mathrm{a}$ and $\mathrm{b}$ are close to zero for this set of tilt angles, which means that the cubic polynomial fitting is near to linear fitting. But in the actual baseline drawing, it did not always happen, and cubic polynomial equation obtained is friendly to the control.

\subsection{Piecewise polynomial fitting}

However, the ground level error in construction site is complex and the fitting error of direct polynomial fitting method may be large for the highgrade baseline. As mentioned before, the computation of coefficients of five-order polynomial fitting method and the control of the MoMaCo is difficult. Therefore, piecewise polynomial fitting method is used [24].

The flow of piecewise polynomial fitting is shown in Figure 7. Firstly, the segmentation is carried out with every four adjacent points. Then, the polynomial fitting is used with each segmentation, and the coefficients of cubic polynomial function can be determined by least squares method. When the last segmentation includes less than four points, the linear or parabola fitting will be carried out.

The expression of each cubic polynomial curve can be defined as

$$
\mathrm{y}_{\mathrm{i}}=\mathrm{a}_{\mathrm{i}} \mathrm{x}^{3}+\mathrm{b}_{\mathrm{i}} \mathrm{x}^{2}+\mathrm{c}_{\mathrm{i}} \mathrm{x}+\mathrm{d}_{\mathrm{i}, \mathrm{i}}=1_{s, \ldots, n} \mathrm{n}
$$

According to the principle of least squares, Eq. (9) can be defined as

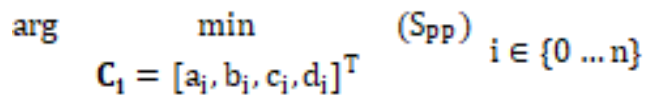

Where

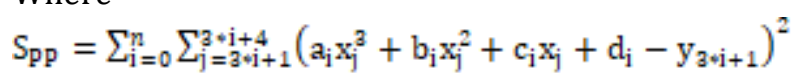

. Then taking the partial derivative with respect to each coefficient in Eq. (9).

$$
\frac{\partial S_{P P}}{\partial a_{\mathrm{i}}}=0, \frac{\partial S_{P P}}{\partial b_{\mathrm{i}}}=0, \frac{\partial S_{P P}}{\partial c_{\mathrm{i}}}=0, \frac{\partial S_{\mathrm{PP}}}{\partial \mathrm{d}_{\mathrm{i}}}=0, \mathrm{i}=0,1_{, \ldots, n} \mathrm{n}(10
$$

$\mathrm{C}_{1}$ can be obtained using the method presented in Sec. 4.2 as well. Corresponding to the data listed in Tab. 1, the result of $\mathbf{C}_{\mathbf{1}}$ can be obtained with Eq. (10) and listed in Table 2. 


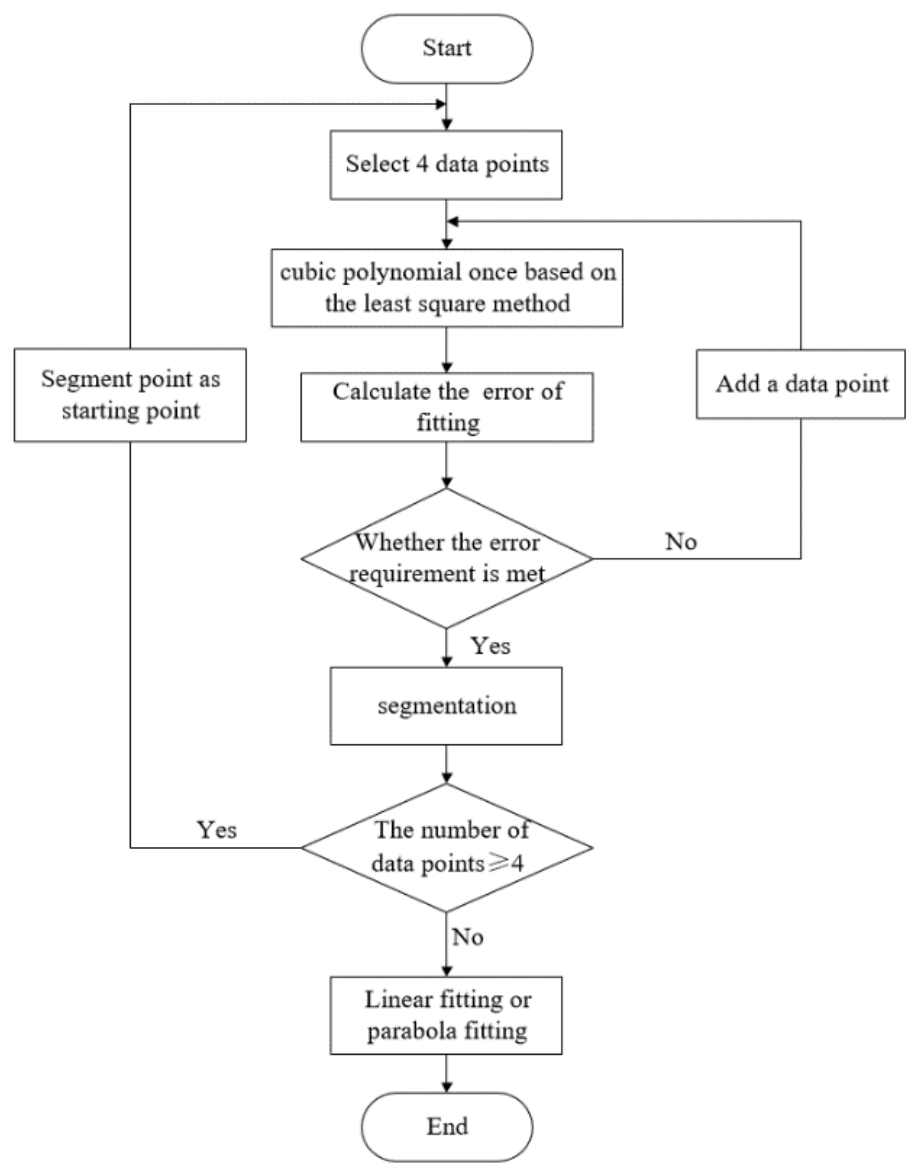

Figure 7: Flow chart of piecewise cubic polynomial fitting

Table 2. The result of piecewise fitting

\begin{tabular}{|c|c|}
\hline $\mathrm{C}_{1}$ & {$[-4.13 \mathrm{e}-08,2.03 \mathrm{e}-05,0.0056,5.14 \mathrm{e}-15]^{\mathrm{T}}$} \\
\hline $\mathrm{C}_{2}$ & {$[-7.24 \mathrm{e}-08,1.53 \mathrm{e}-04,-0.11,24.19]^{\mathrm{T}}$} \\
\hline $\mathrm{C}_{3}$ & {$[-5.94 \mathrm{e}-08,2.07 \mathrm{e}-04,-0.241,87.12]^{\mathrm{T}}$} \\
\hline $\mathrm{C}_{4}$ & {$[1.30 \mathrm{e}-07,-6.17 \mathrm{e}-04,0.96,-4.95 \mathrm{e}+02]^{\mathrm{T}}$} \\
\hline
\end{tabular}

As shown in Figure 8, four cubic polynomial curves are shown in Figure 8.
The maximum fitting error occurs while the length was $1,419 \mathrm{~mm}$.

At this point the fitting error was $0.33 \mathrm{~mm}$ which can meet the need of fitting error of high-grade baseline. It can be found the piecewise fitting method is better than direct fitting method with less fitting error, but it needs more computation resource. In the actual baseline drawing, the method chosen depends on the type of baseline.

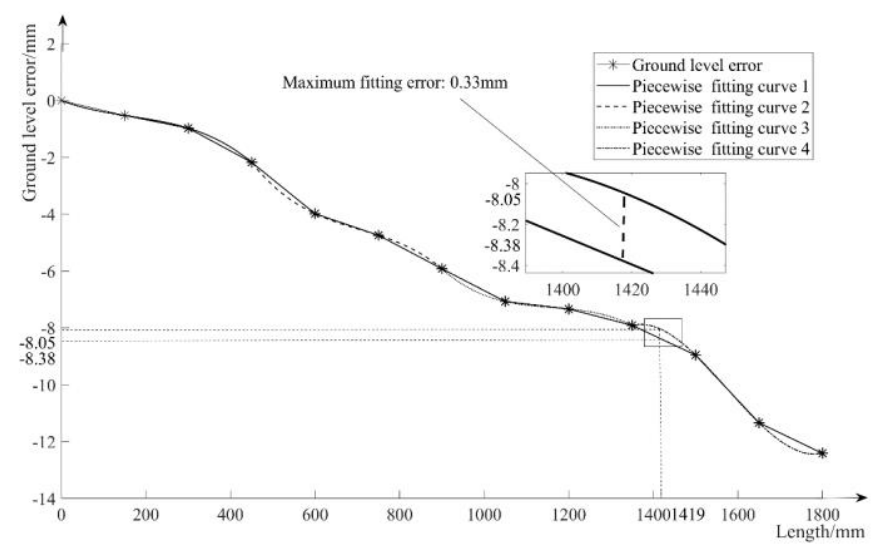

Figure 8: Piecewise Cubic Polynomial Fitting 


\subsection{Compensation with Spatial Movement of MoMaCo}

As mentioned before, the ground level error can be fitting as a cubic polynomial curve or piecewise cubic polynomial curves depending on the type of baseline. The compensation curves are the combination of target baseline with the mirror of the ground level error curve (as shown in Figure 9). Usually, the baselines are straight lines, usually vertical/horizontal lines as shown in Figure 1 (b), so the compensation curves are still cubic polynomial curve. This character makes the compensation curves can be trajectory planned numerically. The planning can be achieved with Eq. (1).

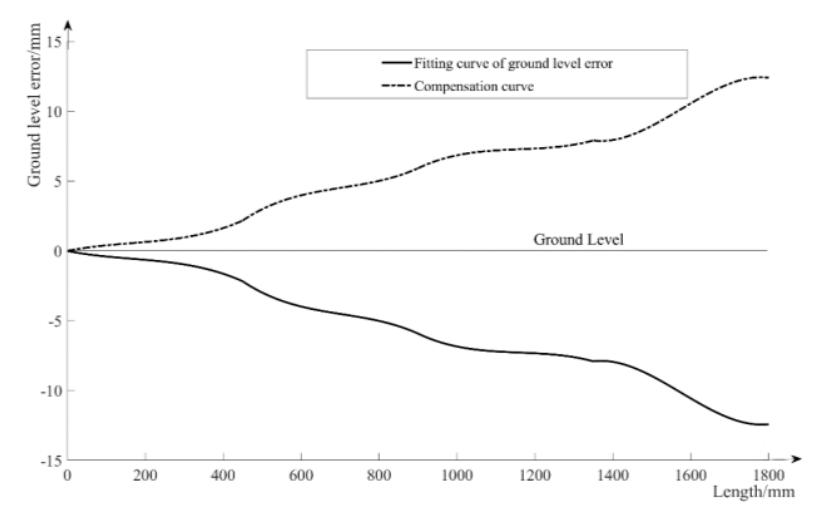

Figure 9: The Compensation Curve

\section{Experiment}

The modeling and compensation method presented in this study is validated using MoMaCo developed by Shanghai University. As shown in Fig. 10, for the experiment, a laser levels is placed in the indoor environment for contrasting.

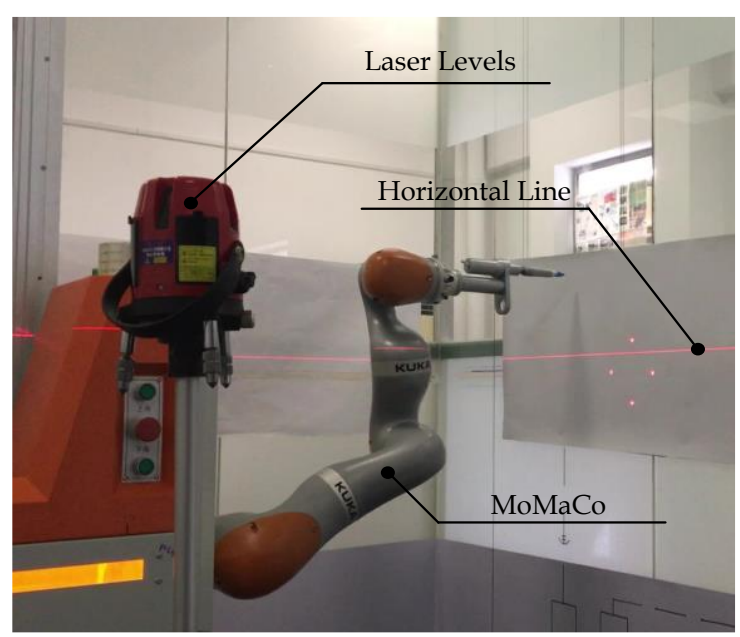

Figure 10: Experiment setup
As shown in Figure 11, the baseline was drawn without ground level error compensated by MoMaCo. This baseline is not horizontal for the ground level error. The corresponding tilt angles are listed in Table 1. The error is obvious in Figure 11 because the red line is projected by laser levels. In Figure 12, the modeling and compensation method were carried out and a new baseline was drawn with compensation. The result is better than the one without compensation. The experiment conducted confirmed these theoretical findings.

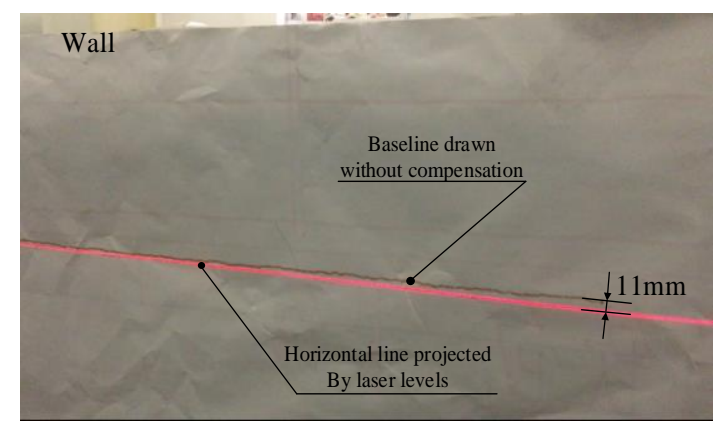

Figure 11: Baseline drawn without compensation

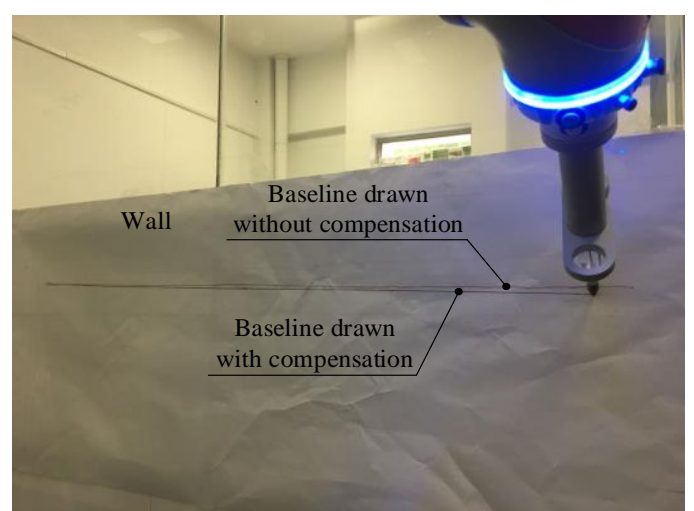

Figure 12: Baseline drawn with compensation

\section{Conclusion}

In this study, a complete method is developed to model and compensate the ground level error in baseline drawing for MoMaCo. A spatial kinematics model of MoMaCo is built. The direct polynomial fitting method and piecewise polynomial fitting method are proposed for common and high-grade baseline respectively. The corresponding fitting errors can be controlled under 1 and $0.5 \mathrm{~mm}$. The compensation method is also presented. The experiment was conducted and the result showed that the modeling and compensation of ground level error is valid to solve this issue.

\section{Acknowledgements}

This work was supported by the National Natural Science Foundation of China (No. 61803251). 


\section{Reference}

[1] Guo S, Song T, Xi FF, and Mohamed RP, "Tip-over stability analysis for a wheeled mobile manipulator," Journal of Dynamic Systems Measurement \& Control, 139(5): 054501-1054501-7, 2017.

[2] Song T, Xi FF, Guo S, and Lin YW, "Optimization of a mobile platform for a wheeled manipulator," Journal of Mechanisms \& Robotics 8(6): 061007 $1-061007-14,2016$.

[3] Song T, Xi FF, Guo S, Tu XW, and Li XY, "Slip analysis for a wheeled mobile manipulator," Journal of Dynamic Systems Measurement \& Control 140(2): 021005-1-021005-12, 2017.

[4] Markus G, Timothy S, Kathrin D, Ian B, Mark B, Gonzalo R, Matthias K, Fabio G, and Jonas B, “ Mobile robotic fabrication at 1:1 scale: the In situ Fabricator," Construction Robotics, vol. 1, pp. 3$14,2017$.

[5] Tim D, Sami Charaf E, Jean-Christophe F, Tobias H, Francesco B, Burkhard C, Roberto G, Fabian H, Bruno L, and Daniel M, "Bots2ReC: introducing mobile robotic units on construction sites for asbestos rehabilitation," Construction Robotics, vol. 1, pp. 29-37, 2017.

[6] Jean-Baptiste I, Alexandre D, Pierre-Elie $H$, Edouard C, David C, Mariola R, and Mikel B, "Large-scale 3D printing with cable-driven parallel robots," Construction Robotics, vol. 1, pp. 69-76, 2017.

[7] Konstantinos L, Anastasios D. D, Christos S, Eftychios P, Konstantinos M, Nikolaos D. D, Angelos A, Philippe C, and Juan V, "Autonomous robotic system for tunnel structural inspection and assessment," International Journal of Intelligent Robotics and Applications, vol. 2, pp. 43-66, 2018.

[8] Steven J, Keating, Julian C, Leland, Levi Cai, and Neri Oxman, "Toward site-specific and selfsufficient robotic fabrication on architectural scales," Science Robotics, 2017.

[9] Guo S, Fang TT, Song T, Xi FF, Wei BG, "Tracking and localization for omni-directional mobile industrial robot using reflectors," Advances in Manufacturing, 6(1):118-125, 2018.

[10] Shuai Guo, Yi Jin, Sheng Bao, and Fengfeng Xi, "Accuracy Analysis of Omnidirectional Mobile Manipulator with Mecanum Wheels," Advances in Manufacturing, vol. 4, pp. 363-370, 2016.

[11] Y.Y. Hsu, and S.S. Wang, "A new compensation method for geometry errors of five-axis machine tools," International Journal of Machine Tools and Manufacture, vol. 47, pp. 352-360, 2007.

[12] F. Xi, Y. Liu, and H.-Y. Feng, "Error Compensations for Three-Dimensional Line Laser Scanning Data," The International Journal of Advanced Manufacturing Technology, vol. 18, pp. 211-216, 2011.

[13] Myeong-Woo Cho, Gun-Hee Kim, Tae-II Seo, Yeon-Chan Hong, and Harry H Cheng, Integrated machining error compensation method using OMM data and modified PNN algorithm," International Journal of Machine Tools and Manufacture, vol. 46, pp. 1417-1427, 2006.

[14] Jinhyun Kim, Wan Kyun Chung, Youngil Youm, and B.H. Lee, "Real-time ZMP compensation method using null motion for mobile manipulators," Proceedings 2002 IEEE International Conference on Robotics and Automation, pp. 1967-1972, 2002.

[15] N.A.Duffie S.J.Malmberg, "Error Diagnosis and Compensation Using Kinematic Models and Position Error Data," CIRP Annals, vol. 36, pp. 355-358, 1987.

[16] Changhai $\mathrm{Ru}$, and Lining Sun, "Improving positioning accuracy of piezoelectric actuators by feedforward hysteresis compensation based on a new mathematical model," Review of Scientific Instruments, vol. 76, pp. 095111-1-8, 2005.

[17] Jianjun Wang, Hui Zhang, and Thomas Fuhlbrigge, " Improving machining accuracy with robot deformation compensation," 2009 IEEE/RSJ International Conference on Intelligent Robots and Systems, pp. 3826-2831, 2009.

[18] Chung-Lin Huang, and Chao-Yuen Hsu, "A new motion compensation method for image sequence coding using hierarchical grid interpolation," IEEE Transactions on Circuits and Systems for Video Technology, vol. 4, pp. 42-52, 1994.

[19] Xinda Song, Jiancheng Fang, Bangcheng Han, and Shiqiang Zheng, "Adaptive Compensation Method for High-speed Surface PMSM Sensorless Drives of EM Position Estimation Error," IEEE TRANSACTIONS ON POWER ELECTRONICS, vol. 31, no. 2, pp. 1438-1449, 2016.

[20] John M.Fines, and ArvinAgah, "Machine tool positioning error compensation using artificial neural networks," Engineering Applications of Artificial Intelligence, vol. 21, pp. 1013-1026, 2007.

[21] Takaaki Oiwa, "Error Compensation System for Joints, Links and Machine Frame of Parallel Kinematics Machines," The International Journal of Robotics Research, vol. 24, pp. 1087-1102, 2005.

[22] Hamid Taheri, Bing Qiao, and Nurallah Ghaeminezhad, "Kinematic Model of a Four Mecanum Wheeled Mobile Robot. International Journal of Computer Applications," vol. 113, no. 3, pp. 6-9, 2015.

[23] Yifan Jia, Xiaodong Song, and Sendren ShengDong $\mathrm{Xu}$, "Modeling and motion analysis of fourMecanum wheel omni-directional mobile platform," 2013 CACS International Automatic Control Conference, pp. 328-333, 2014.

[24] Kozo Ichida, Takeshi Kiyono, and Fujiichi Yoshimoto, "Curve Fitting by a One-Pass Method With a Piecewise Cubic Polynomial," ACM Transactions on Mathematical Software, vol. 3, pp. 164-174, 1977. 